\title{
The role of electromagnetism in tidal disruption events
}

\author{
Andrej Čadež \\ University of Ljubljana \\ email: andrej.cadez@fmf.uni-lj.si
}

\begin{abstract}
Tidal disruption events are characterized by the prompt release of very high energy electromagnetic radiation, which rapidly carries away a significant fraction of mass-energy. The time scale od prompt release is quite comparable to the time scale of gravitational collapse. We suggest that generation of very high energy radiation from pulsar nebulae may be an example of the relativistic coupling between gravity and electromagnetism. The main ingredient of our picture comes from the observation that the electron - ion energy exchange time scale is much longer than the electron ion energy exchange time scale, which leads to thermodynamic decoupling of electron and ion gases, and thus to their different temperatures. Under such conditions the dominating hotter component pushes the colder component further out and thus generates a global electric field which constrains electrons and ions by their mutually generated electric field, yet allows them to reach very high kinetic energies
\end{abstract}

Keywords. plasmas, relativity, cosmic rays

\section{Introduction}

High energy phenomena in astrophysics take many forms, from gamma ray bursts exploding in high energy jets, to steady accretion X-ray emitting disks and finally to plerionic nebulae, such as Crab, which emit gamma rays at $\mathrm{TeV}$ energies. The emission of a significant portion of energy by a relatively small number of photons, which require a relatively small number of very high energy charged particles to produce, is the common characteristic of all these phenomena. Jets are another common trait. Since the Crab nebula and its pulsar have been studied extensively, providing a wealth of data on its behaviour, it might be of interest to discuss the many chalenges that these data present.

A closely studied property of the Crab pulsar is the history of its slow down by the Jodrell Bank Radio Observatory. After 23 years of monitoring Lyne et al.(1993) were able to state: "Between glitches, the rotational slowdown of the Crab pulsar is well described by a power law with braking index $2.51 \pm 0.01$.", while 22 years later the same authors (Lyne et al. (2015)) came to the conclusion: "Overall, a low mean braking index of 2.342(1) is measured for the whole period, compared with values close to 2.5 in intervals between glitches". Using the published Jodrell Bank data since 1988, together with insight from scarce but much more precise optical observations, Cadež et al. (2016) come to a different conclusion. Instead of analyzing the frequency and frequency derivative behaviour, they represent the rotational phase of the pulsar during the mentioned period by ten episodes delimited by nine mayor glitches. Each episode, lasting a few years, can be fitted to a specific braking index law, with the braking index taking values between $\sim 2.2$ and $\sim 2.6$. The phase difference from the pure braking index law always follows a simple relaxation decay with an amplitude no larger than 17 turns $\dagger$. This result is interpreted as a hint

$\dagger$ With the total phase change during the episode of $3 \times 10^{9}$ turns. 
that differences between braking episodes are caused by abrupt changes in electromagnetic coupling of the rotating magnetic field of the pulsar to the surrounding nebula. Considering the relaxation time scales and the durations of episodes, we explore this as a hint that coupling to the braking mechanism is caused by changes in the structure of the surrounding nebula rather than in the interior structure of the pulsar. This interpretation eventually relates to other observed, yet unexplained phenomena such as the formation of a global magnetic field, production of extremely high energy radiation, formation of jets, accelerated expansion of the nebula etc.

\section{How to heat the nebula?}

Let us consider the pulsar as a generator of a rotating electromagnetic field at the center of the nebula, a medium with a certain permittivity $\varepsilon$ and conductivity $\sigma$. The propagation of electromagnetic wave in such a medium is characterized by the dispersion relation $k^{2}=\varepsilon\left(\frac{\omega}{c}\right)^{2}+i \mu_{0} \sigma \omega$, where omega is the angular frequency of the propagating wave - the angular rotation frequency of the pulsar in our case. The solution of Maxwell's equations can be expanded in multipoles of damped propagating waves, which dissipate energy along the way. Considering the solution which at $r=r_{p}$ limits to a rotating magnetic dipole $\left(\left|k r_{p}\right| \ll 1\right)$ with a dipole moment $p_{m}$, we find the following expressions for the energy outflow through the sphere with radius $r_{p}$ :

$$
\begin{array}{rlrl}
\varepsilon>0: L=\frac{\mu_{0}}{6 \pi} \omega^{2}\left(\varepsilon^{3 / 2} \frac{\omega^{2}}{c^{3}}+\frac{\mu_{0} \sigma}{r_{p}}\right) p_{m}^{2} & n=3-\frac{2}{1+\varepsilon^{3 / 2}\left(\frac{\omega}{c}\right)^{2} \frac{r_{p}}{\mu_{0} \sigma c}} \\
\varepsilon<0: L=\frac{\mu_{0}}{6 \pi} \omega^{2} \frac{\mu_{0} \sigma}{r_{p}} p_{m}^{2} & n=1
\end{array}
$$

Considering that energy outflow is fed by rotational energy of the pulsar $\left(\frac{d}{d t}\left(\frac{1}{2} J \omega^{2}\right)=\right.$ $-L)$, one can calculate the corresponding braking index $n=\frac{\ddot{\omega} \omega}{\dot{\omega}^{2}}$, which is also listed above. This simple model explains the average observed braking index $n \sim 2.5$ if $\varepsilon>0$ and $\sigma=\frac{1}{3} \varepsilon^{3 / 2}\left(\frac{\omega}{c}\right)^{2} \frac{r_{s}}{\mu_{0} c}=3.5 \times 10^{-12}\left[\frac{A}{V m}\right] \varepsilon^{3 / 2}$. On first sight both conditions seem difficult to satisfy: 1 . considering the often quoted electron density $\sim 10^{3} \mathrm{~cm}^{-3}$, one finds that the plasma frequency should be a few $\mathrm{kHz}$, which would imply that below a few $\mathrm{kHz} \varepsilon$ should be negative. 2. Even if the value of $\varepsilon$ should be found positive, the required plasma conductivity is unusually low. Yet, the conditions at the center of the Crab nebula may be sufficiently unusual to allow both obstacles to be surmounted. First consider the conductivity: $\sigma=\frac{n_{e} e^{2}}{m_{e} \nu_{c}}$, where $\nu_{c}$ is the collision frequency of an electron with all other particles and fields present. Because of the evidently low density of plasma in question, electron-electron, electron-ion and electron-atom collision frequencies are many orders of magnitude too low to satisfy our condition, but the remaining candidate - the exchange of energy-momentum with electromagnetic field through synchrotron radiation - can do the job. So we derive the electron collision frequency $\nu_{c}$ as the inverse of synchrotron lifetime to write: $\nu_{c}=\frac{\sigma_{T}}{m_{e} c} \frac{B^{2}}{\mu_{0}} \gamma_{e}$, where $\sigma_{T}$ is the Thomson cross section, $B$ the strength of the static component of the magnetic field, and $\gamma_{e}$ the relativistic Lorentz gamma factor of electrons. When this expression is used to calculate the conducivity inserted in eq.(2.1), one obtains the following expression for the braking power: $L=\int \sigma_{T} c \gamma_{e}^{2} n_{e} \frac{B^{2}}{\mu_{0}} d V \approx$ $2\left\langle\gamma_{e}{ }^{2} n_{e}\right\rangle \sigma_{T} c \int \frac{B^{2}}{2 \mu_{0}} d V$. Here \langle\rangle stands for the suitable average over the volume with high magnetic field near the pulsar; the magnetic energy, represented by the above integral, is taken to be the energy belonging to a static magnetic dipole with a strength $10^{8} \mathrm{~T}$ at the surface of the pulsar (at $r_{p}=10 \mathrm{~km}$ ). In this way the braking power becomes 
$L=3.3 \times 10^{38}[\mathrm{erg} / \mathrm{s}] \frac{n_{e}}{1000 \mathrm{~cm}^{-3}}\left(\frac{\gamma_{e}}{10^{4}}\right)^{2}$, which should be compared to the invoked slow down luminosity $\left(L_{C r a b} \sim 2 \times 10^{38}[\mathrm{erg} / \mathrm{s}]\right)$. If one further relates the high energy peak at $\sim 10^{12} \mathrm{eV}$ in the energy spectrum of the Crab (Kohri et al. (2012)) to the energy of electrons in the heating region, one comes to the conclusion that extremely low electron density in the central region suffices to couple pulsar rotation to the nebula. However, it is quite clear that pulsar's gravitational field can not bind plasma of correspondingly high temperature to the region where the electromagnetic field can heat it. Thus, this proposal can only work if large scale electric and magnetic fields can provide suitable potential wells to sustain the conversion of energy in the central heating region.

We start with a very simple scenario, considering the central $7 \times 10^{5} \mathrm{~km}$ region as the heater where at least electrons can be accelerated to very high kinetetic energy by the central electromagnetic source (the pulsar). The heater is surrounded by a tenuous high energy synchrotron nebula, visible almost exclusively in X-rays (Chandra image), which merges with the ejecta shell at the distance $R$ roughly 1 pc from the pulsar. The electronion energy echange in the synchrotron nebula is extremely slow - of order millions of years. Thus, no mechanism within the nebula enforces equipartition of energy between electrons and ions. The average energy of electrons is regulated mainly by the balance of energy input from the heater and cooling by synchrotron radiation. Ions are only weakly coupled to radiation but couple thermaly to the relatively cold ejecta boundary. Thus we expect electrons in the synchrotron nebula to have higher kinetic temperature than ions. The phase space distribution of constituents of both gassses can be described by Boltzmann distribution functions $f_{e}(\vec{r}, \vec{p})=e^{-\alpha_{e}-\beta_{e} H_{-}}$and $f_{i}(\vec{r}, \vec{p})=e^{-\alpha_{i}-\beta_{+} H_{+}}$, where the single particle Hamiltonians $H_{ \pm}=\alpha(r) \sqrt{m_{ \pm}^{2} c^{2} / \psi^{4}(r)+m_{ \pm}^{2} c^{2}} \pm e \phi(\vec{r})$ govern the motion of electrons ("-") and ions ("+") in the gravitational field of the pulsar and the electric field they generate. Here $\alpha(r)=\left(1-\frac{M^{*}}{2 r}\right) /\left(1+\frac{M^{*}}{2 r}\right)$ and $\psi=1+\frac{M^{*}}{2 r}$ are the relevant Schwarzschild metric coefficients in isotropic coordinates, $M^{*}=G M_{p} / c^{2}$ is the gravitational radius of the pulsar and $\phi(\vec{r})$ is the electric potential generated by the charge distribution. The Lagrange multipliers $\alpha_{e}, \alpha_{i}, \beta_{e}$ and $\beta_{i}$ are determined by the numbers and energies of ions and electrons. Introducing $\tau_{e}=m_{e} c^{2} / \beta_{e}, \tau_{i}=m_{p} c^{2} / \beta_{i}, F(\vec{r})=$ $\frac{e \phi(\vec{r})}{m_{e} c^{2} \tau_{e}}, \tilde{F}(\vec{r})=\frac{e \phi(\vec{r})}{m_{e} c^{2} \tau_{p}}, \tilde{\alpha}(r)=m_{i} \alpha(r) / m_{e}, B_{n}(x)=e^{x} \operatorname{Bessel} K[n, x]$ and denoting Compton wavelength as $\lambda_{c}$ and classical electron radius as $r_{c l}$, these can be written as :

$$
\begin{aligned}
N_{e} & =\int \frac{4 \pi}{\lambda_{c}^{3} \alpha} \tau_{e} e^{F-\alpha_{e}} B_{2}\left(\frac{\alpha}{\tau_{e}}\right) d V \\
N_{i} & =\int \frac{4 \pi}{\lambda_{c}^{3} \tilde{\alpha}} \tau_{i} e^{\tilde{F}-\alpha_{e}}\left(\frac{m_{p}}{m_{e}}\right)^{2} B_{2}\left(\frac{\tilde{\alpha}}{\tau_{e}}\right) d V \\
E_{e} & =\int \frac{m_{e} c^{2}}{\lambda_{c}^{3} \alpha^{2}} e^{F-\alpha_{e}} \tau_{e}^{3}\left[(3-F) \frac{\alpha}{\tau_{e}} B_{0}\left(\frac{\alpha}{\tau_{e}}\right)+\left(6-2 F+\frac{\alpha^{2}}{\tau_{e}^{2}}\right) B_{1}\left(\frac{\alpha}{\tau_{e}}\right)\right] d V \\
E_{i} & =\int \frac{m_{p} c^{2}}{\lambda_{c}^{3} \tilde{\alpha}^{2}} e^{\tilde{F}-\alpha_{e}} \tau_{e}^{3} \frac{m_{p}}{m_{e}}\left[(3-\tilde{F}) \frac{\tilde{\alpha}}{\tau_{p}} B_{0}\left(\frac{\tilde{\alpha}}{\tau_{p}}\right)+\left(6-2 \tilde{F}+\frac{\tilde{\alpha}^{2}}{\tau_{p}^{2}}\right) B_{1}\left(\frac{\tilde{\alpha}}{\tau_{p}}\right)\right] d V
\end{aligned}
$$

Here $d V=4 \pi r^{2} \psi^{6}(r) d r$ and the electric potential equation turns into:

$$
F^{\prime \prime}(r)+\frac{2}{r \psi(r)} F^{\prime}(r)=-16 \pi^{2} \frac{r_{c l}}{\lambda_{c}^{3}} \frac{\psi^{4}(r)}{\alpha(r)}\left[-e^{F-\alpha_{e}} B_{2}\left(\frac{\alpha(r)}{\tau_{e}}\right)+\frac{m_{p}^{2} \tau_{i}}{m_{e}^{2} \tau_{e}} e^{-\tilde{F}-\alpha_{i}} B_{2}\left(\frac{\alpha(r) m_{i}}{m_{e} \tau_{i}}\right)\right],
$$

with boundary conditions $N_{e}=N_{i}$ and $F\left(r_{e j}\right)=0$ at the outer boundary of ejecta shell. An insight into eq.(2.7) can be obtained from the low energy $\left(\tau_{e}, \tau_{i} \ll 1\right)$, low gravity 
$\left(r_{p} \gg M^{*}\right), \tau_{e} \neq \tau_{i}$ solution $F(r) \approx \frac{15}{8}\left(\frac{M^{*}}{r}-\frac{M^{*}}{r_{e j}}\right)\left(\frac{1}{\tau_{i}}-\frac{1}{\tau_{e}}\right)^{-1}+\mathcal{O}\left(\frac{M^{* 2}}{r^{2}}\right)$, which makes the right hand side of eq.(2.7) vanish $\dagger$, while the remainder is $\mathcal{O}\left(M^{* 2} / r^{4}\right)$. The star and the ejecta shell act like a spherical capacitor charged with the hotter componet at the outer electrode. The potential difference $U=\frac{m_{e} c^{2}}{e} \tau_{e}\left(F\left(r_{e j}\right)-F\left(r_{s}\right)\right)$ is obviously quite small in the nonrelativistic limit. In higly relativistic case equation (2.7) must be solved numerically. Solutions $F(r)$ again only slightly differ from the capacitor field form $F \approx \frac{M^{*} q}{r}$, only the value of $q$ must be determined numerically. In the regime $10^{4}<\tau_{e}<5 \times 10^{4}$, which corresponds to electrons capable of emitting at $\sim 10^{12} \mathrm{eV}$, characteristic of Crab high energy radiation, and $\tau_{i} \sim 1300, q$ is found to have an almost constant value $q \approx 21$. With this solution one can go back to the braking power calculation and refine it by using the radial dependence of electron number density, expressed as $n_{e}=n_{0} e^{q M^{*} / r}$, to better evaluate the volume integral. Neglecting the dependence of $\alpha$ and $\psi$ on $r$, realizing that $\gamma_{e}=3 \tau_{e}$ and defining $x=\frac{M^{*} q}{r_{p}}=3.15$, the result can be written in the form

$$
\begin{aligned}
L & =27 \times 3.3 \times 10^{35}[\mathrm{erg} / \mathrm{s}]\left(\frac{n_{e}}{\mathrm{~cm}^{-3}}\right)\left(\frac{\gamma_{e}}{10^{4}}\right)^{2}\left[e^{x}\left(\frac{1}{x}-\frac{2}{x^{2}}+\frac{2}{x^{3}}\right)-2 \frac{1}{x^{2}}\right] \\
& =3.7 \times 10^{37}[\mathrm{erg} / \mathrm{s}]\left(\frac{n_{e}}{\mathrm{~cm}^{-3}}\right)\left(\frac{\tau_{e}}{10^{4}}\right)^{2}=0.18 L_{C r a b}\left(\frac{n_{e}}{\mathrm{~cm}^{-3}}\right)\left(\frac{\tau_{e}}{10^{4}}\right)^{2} .
\end{aligned}
$$

\section{Conclusion}

We have identified the tenuous region between the shock front and the compact remnant of a supernova-like explosion as a region where gravity and electromagnetism strongly couple through tenuous relativistic plasma. Applying these ideas to the tenuous plasma of inner Crab nebula, equations (2.2) and (2.8) plausibly connect pulsar braking power to high electron temperature and low density of the inner nebula. In the proposed mechanism gravity and electromagnetism couple directly, since the main component of the electric field is produced by charges on the capacitor between the central compact object and outer ejecta layer. The plasma inside the capacitor does not importantly contribute to electric field by polarization (as long as it is collisionless), however the polarization is sufficient to concentrate the hotter component (electrons) in the inner region and deplete the colder component there. This eliminates the plasma frequency limit in the inner region, and allows electrons to couple strongly and rapidly to electromagnetic field of the central object. This may be the the fastest way to turn electromagnetic or gravitational energy transfer into observable radiation.

\section{References}

Lyne, A. G., Pritchard, R. S., \& Graham-Smith, F. 1993, MNRAS, 265, 1003

Lyne, A. G., Jordan, C. A., Graham-Smith, F., et al. 2015, MNRAS, 446, 857

Čadež, A., Zampieri, L., Barbieri, C., et al. 2016, A\&A, 587, A99

Kohri, K., Ohira, Y., \& Ioka, K. 2012, MNRAS, 424, 2249

$\dagger$ It is worth noting that the right hand side of equation (2.7) does not vanish for $F=0$, only because $B_{2}(x)$ deviates from a pure power law. In the classical nonrelativistic treatment $B_{2}\left(\frac{\alpha(r)}{\tau_{e}}\right)$ would be replaced by $\sqrt{\frac{2 \pi \tau_{e}}{\alpha(r)}}$ and similarly $B_{2}\left(\frac{\alpha(r) m_{i}}{m_{e} \tau_{e}}\right)$. In this case $\alpha^{-1 / 2}(r)$ would be the common factor of the two terms on the right hand side of eq.(2.7), which could be made to vanish for $F=0$ at all $r$ with a proper choice of $\alpha_{i}$ and $\alpha_{e}$. Thus, the emergence of electric field should be considered as a relativistic effect. 